\title{
COSMIC RAY STUDIES WITH AN INTERSTELLAR PROBE
}

\author{
R. A. Mewaldt and E. C. Stone \\ California Institute of Technology, Pasadena, CA 91125
}

\begin{abstract}
Among the NASA mission concepts that have been suggested for the 21st century is an Interstellar Probe that might be accelerated to a velocity of $\sim 10$ to $20 \mathrm{AU} / \mathrm{yI}$, allowing it to leave the heliosphere, ultimately reaching a radial distance of $\sim 500$ to $1000 \mathrm{AU}$ in $\sim 50$ years. We discuss briefly previous studies of such a mission, and its potential significance for cosmic ray studies, both within the heliosphere, and beyond, in interstellar space.
\end{abstract}

\section{INTRODUCTION}

The recent report ${ }^{1}$ Space Science in the 21st Century included discussion of an "Interstellar Probe", which might be launched sometime after the year 2000, reaching a radial distance of $100 \mathrm{AU}$ within 5 to 10 years. With a velocity of escape from the solar system of $\sim 50$ to $100 \mathrm{~km} / \mathrm{sec}$ a distance of 500 to $1000 \mathrm{AU}$ could be reached in an extended mission of $\sim 50$ years. The acceleration required for the necessary spacecraft velocity might be achieved by one of several possible schemes ${ }^{2}$, including Jupiter gravity assist and subsequent swingby of the Sun at $\sim 4$ solar radii, or the use of megawatt nuclear electric propulsion. Data could, if necessary, be transmitted to Earth with advanced optical communications systems. For example, a 1-m optical telescope combined with a 10-W laser transponder can transmit $20 \mathrm{kbps}$ to a $10-\mathrm{m}$ Earth-orbiț-based telescope from $1000 \mathrm{AU}$. However, conventional systems, such as those on Voyager, are adequate for lower bit rate requirements $(<1 \mathrm{kbps})$.

The Jet Propulsion Laboratory has recently studied a mission called TAU (for Thousand AU), which addresses objectives that cover a broad range of scientific disciplines, including: ${ }^{3}$ optical astronomy and astrometry; cosmology; stellar evolution; gamma-ray astrophysics; gravitation; solar system studies; and space physics including cosmic ray studies. The strawman payload included several energetic particle and cosmic ray detectors, as well as an optical/IR telescope, UV spectrometer, VLF radio astronomy antenna, magnetometer, and instruments to detect plasma and plasma waves, dust, and $\gamma$-ray bursts. Total spacecraft plus payload masses in the range from several hundred to $1000 \mathrm{~kg}$ have been discussed $^{1,2}$.

\section{HELIOSPHERIC AND INTERSTELLAR COSMIC RAY STUDIES}

In our present view of the large-scale structure of the heliosphere (see, e.g., ref. 4), the solar wind (SW) flows radially outward to a termination shock, surrounded at a somewhat greater distance by a contact surface called the "heliopause", the boundary between the SW plasma and the interstellar gas. 


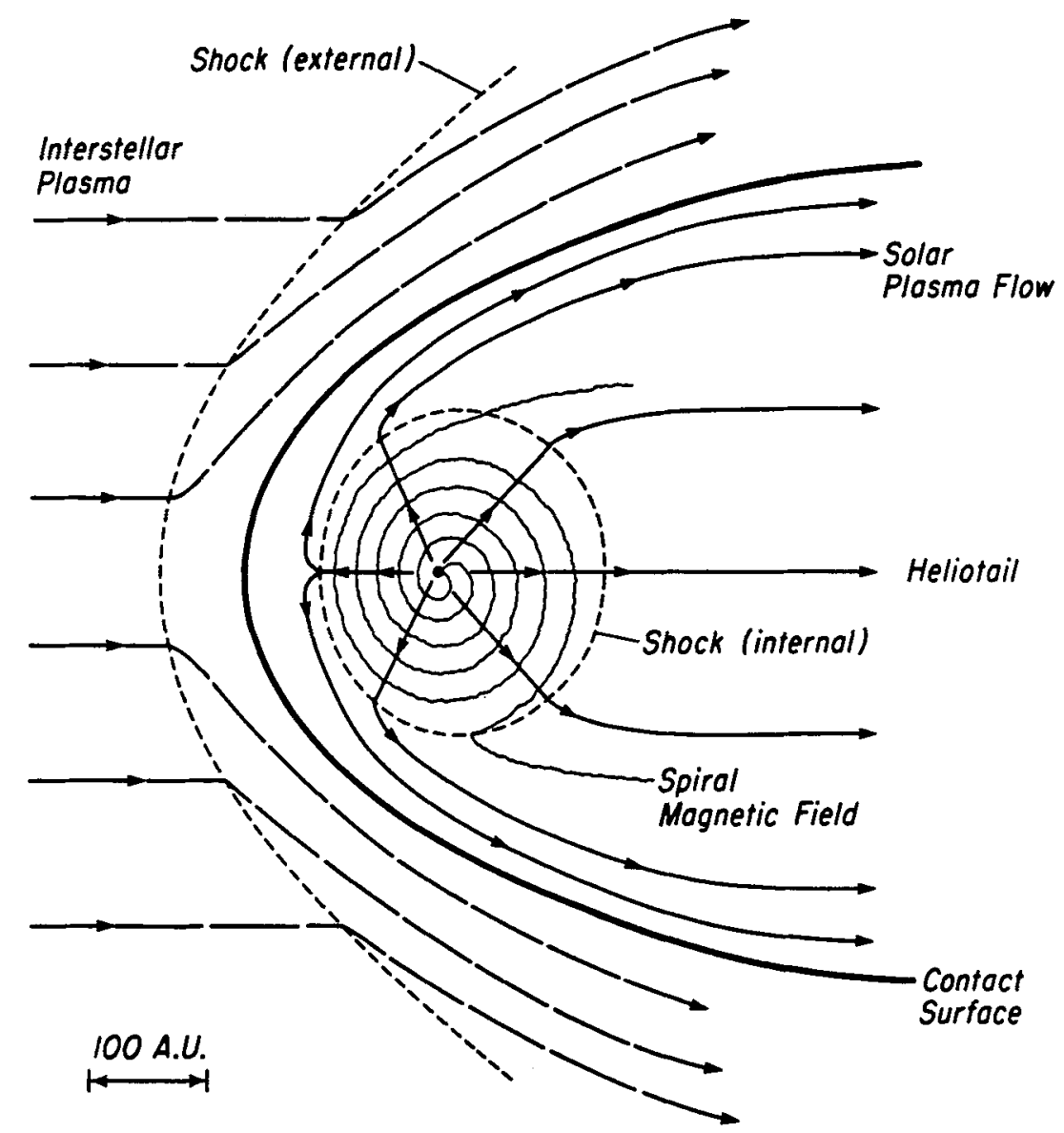

Figure 1: Schematic overview of the heliosphere indicating the termination shock and the contact surface (by J. R. Jokipii; see also ref. 6).

Recent calculations ${ }^{5}$ indicate that the interstellar spectrum of cosmic rays is unmodulated outside of the heliopause, which would then correspond to the cosmic ray "modulation boundary". The termination shock, on the other hand, is the likely site of significant particle acceleration, including the origin of the anomalous cosmic ray component ${ }^{7}$. Holzer ${ }^{4}$ has recently estimated a distance of 50 to $60 \mathrm{AU}$ to the termination shock, with an estimated uncertainty of a factor of $\sim 2$. The heliopause is estimated to be approximately twice as distant as the termination shock. Some indirect estimates of the distance to the cosmic ray modulation boundary are as small as $\sim 50 \mathrm{AU}$ (e.g., refs. 8, 9).

Figure 2 shows the radial distance of Pioneer 10 and Voyagers 1 and 2 from the Sun as a function of time, along with two examples of possible trajectories for an Interstellar Probe. Note that while it is possible that one (or more) of the Voyager or Pioneer spacecraft may cross the termination shock within its operating lifetime, it is much less likely that one will cross the heliopause. 


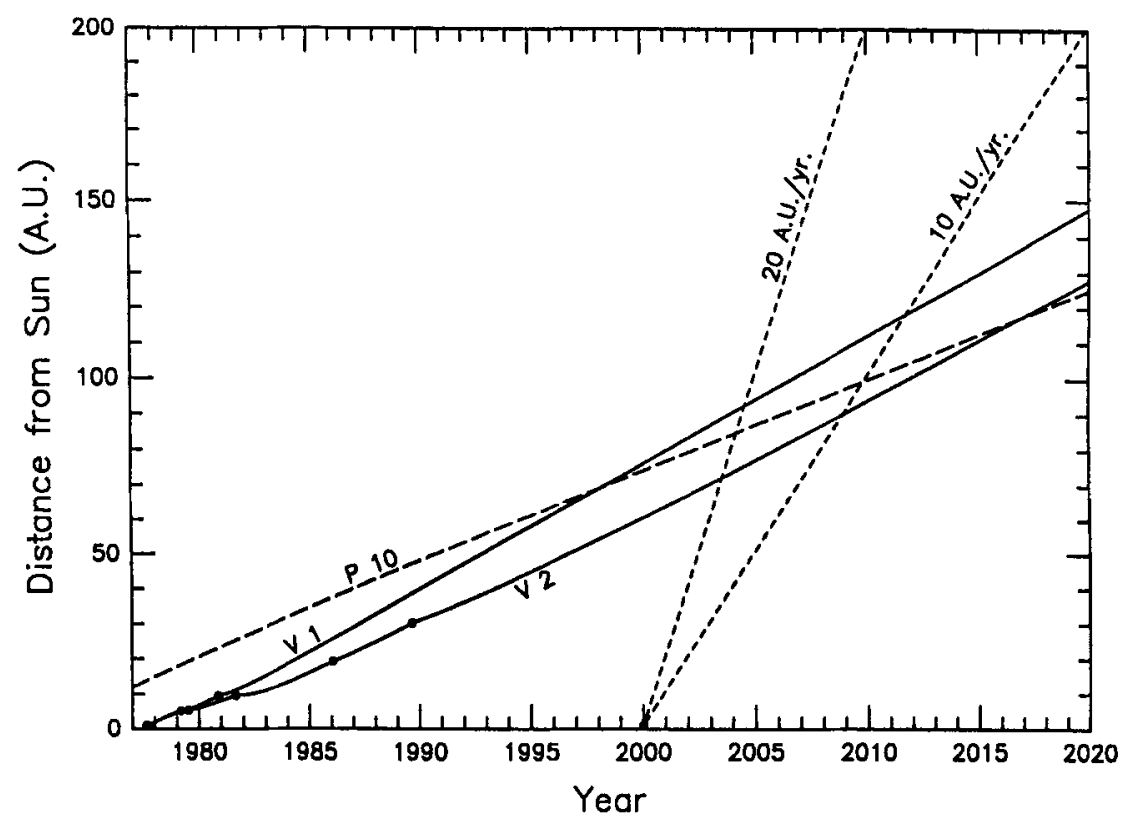

Figure 2: Radial distance of Pioneer 10 and Voyagers 1 and 2 from the Sun as a function of time, along with possible trajectories for an Interstellar Probe. Note that Pioneer 10 is expected to run out of power sometime before the year 2000, while the Voyagers may last until $\sim 2015$. The Voyagers are traveling in the general direction of the front of the heliosphere, while Pioneer-10 is traveling in the opposite direction. Planetary encounters of the Voyagers are indicated.

Cosmic ray science objectives that could be addressed with an Interstellar Probe into the local interstellar medium (ISM) would include the following:

- Measurement of the interstellar spectra of cosmic ray nuclei below $\sim 1 \mathrm{GeV} / n u c$.

- Measurement of the interstellar electron spectrum below $\sim 200 \mathrm{MeV}$.

- Determination of the "primary" and "secondary" contributions to cosmic ray' electrons.

- Detection of $\sim 1 \mathrm{MeV}$ interstellar positrons from ${ }^{56} \mathrm{Co}$ decay in supernovae, the likely source of the galactic $0.511 \mathrm{MeV} \gamma$-ray line.

- In situ study of the acceleration of the anomalous cosmic ray component, including both seed and accelerated nuclei.

- In situ measurement of acceleration effects of the SW termination shock on GCR nuclei and electrons.

- Measurement of spatial gradients of galactic, solar, and interplanetary particles within the heliosphere.

- Correlation of interplanetary cosmic ray, SW, and magnetic field parameters in the outer heliosphere. 
- Identification of the cosmic ray modulation "boundary".

- Measurement of local ISM abundances for comparison with SEP, SW, GCR, ACR, and spectroscopic abundances.

- Determination of the cosmic ray contributions to the "cosmic abundances" of nuclei such as ${ }^{6} \mathrm{Li}$ and ${ }^{7} \mathrm{Li}$, of interest to early universe studies.

- Measurement of interstellar parameters to normalize cosmic ray propagation models.

- Determination of the cosmic ray contribution to the heating, pressure, and energy balance of the local ISM.

- Search for interstellar cosmic ray anisotropies due to nearby/recent supernovae.

An Interstellar Probe admittedly poses significant technical challenges, including development of the propulsion and data systems, and the need for a spacecraft and instruments (not to mention investigator teams) with $\sim 50$ year lifetimes. Because of the broad range of objectives for such a mission, it would be an obvious candidate for cooperation with NASA scientific disciplines outside of Space Physics. While a broad-based mission such as $\mathrm{TAU}^{3}$ would clearly be a very major scientific undertaking, it is also worth considering less ambitious (and less expensive) options that address a subset of these objectives. In the end, mass and power limitations always force some compromises on instrumentation, but it would appear that an Interstellar Probe could address a large fraction of the outstanding problems in cosmic ray physics.

Acknowledgements: This work was supported in part by NASA under grant NGR 05-002-160. We thank M. D. Looper for producing Figure 2 and R. Ramaty for suggestions.

\section{REFERENCES}

1) Space Science in the Twenty-First Century: Imperatives for the Decades 1995 to 2015, Task Group on Solar and Space Physics, National Academy Press, Washington D.C. (1988).

2) Jaffe, L. D. et al., An Interstellar Precursor Mission, JPL Publication 77-70 (1977).

3) Etchegaray, Maria Ines, Preliminary Scientific Rationale for a Voyage to a Thousand Astronomical Units, JPL Publication 87-17 (1987).

4) Holzer, T. E., to appear in Ann. Rev. Astron. Ap. (1989).

5) Jokipii, J. R., as quoted in ref. 6.

6) Mewaldt, R. A., A. C. Cummings, J. H. Adams Jr., P. Evenson, W. Fillius, J. R. Jokipii, R. B. McKibben, and P. A. Robinson Jr., in Interplanetary Particle Environment, Joan Feynman and Stephan Gabriel editors, JPL Publication 88-28, p. 14 (1988).

7) Pesses, M. E., J. R. Jokipii and D. Eichler, Ap. J. (Letters) 246, L85 (1981).

8) Randall, B. A., and J. A. Van Allen, Geophys. Research Lett. 13, 628 (1986).

9) Webber, W. R., Astr. Ap., 179, 277 (1987). 